Pacific Journal of Mathematics

HORROCKS' QUESTION FOR MONOMIALLY GRADED 


\section{HORROCKS' QUESTION FOR MONOMIALLY GRADED MODULES}

\section{LARRY SANTONI}

In this paper several related inequalities are developed which, when appropriately specialized, yield affirmative answers to Horrocks' question in the case of multi-graded $R\left[X_{1}, \ldots, X_{n}\right]$-modules for certain rings $R$.

1. Introduction. In 1978 Hartshorne reported a question [H, Problem 24] due to Horrocks which essentially asks whether the $i$ th Betti number of a finite length module over an $n$-dimensional regular local ring is at least as large as the corresponding binomial coefficient $\left(\begin{array}{l}n \\ i\end{array}\right)$. Recall that the $i$ th Betti number of a module $M$ is defined to be the rank of an $i$ th syzygy of $M$ with respect to a minimal free resolution. Equivalently, if $(R, m, k)$ is a regular local ring, the question asks whether $\operatorname{dim}_{k} \operatorname{Tor}_{i}^{R}(k, M) \geq\left(\begin{array}{c}n \\ i\end{array}\right)$. Evans and Griffith [EG $\mathbf{E}$ ] give an affirmative answer to the conjecture for finite length modules over the polynomial ring $k\left[X_{1}, \ldots, X_{d}\right]$, with $k$ a field, which are direct sums of cyclic modules $k\left[X_{1}, \ldots, X_{d}\right] / I$, where $I$ is generated by monomials.

One of the principal results of this paper is contained in Theorem 3.3 which gives a quite general inequality relating values of an additive function defined on a class $\mathscr{C}$ of $R\left[X_{1}, \ldots, X_{d}\right]$-modules satisfying some very reasonable closure conditions.

Seemingly more stringent conditions must be put on the modules themselves, namely admission of a "high-low decomposition", but at the moment it is unclear just how restrictive this condition is. However the base ring $R$ need only be commutative with identity.

An easy application of Theorem 3.3 extends the result of Evans and Griffith to the larger class of all finite length modules graded by monomials over some regular local (or graded) based ring $R$ for which Horrocks' inequality is known to hold, e.g. $\mathbb{Z}^{d}$-graded over $R\left[X_{1}, \ldots, X_{d}\right]$ with $R$ regular and Krull $\operatorname{dim} R \leq 4$.

In $\S 4$ the techniques used in the proof of Theorem 3.3 are employed in a somewhat different fashion to obtain lower bounds for images of certain maps of Koszul homology. In this instance it will be seen that a high-low decomposition is used in a way quite different than in 
Theorem 3.3, suggesting that this property may be of more importance than simply its utility in this paper.

In $\S 5$, Theorem 3.3 is applied again to produce a remarkable result on iterated Koszul homology. This result is a generalization (and rephrasing in the language of Koszul homology) of a conjecture made by Evans and Griffith about arbitrary finite length modules. The principal result is contained in Theorem 5.2 which specializes to Theorem 5.5:

Let $M$ be a finite length module multi-graded over $k\left[X_{1}, \ldots, X_{d}\right]$ and let $\mathbf{x}_{1}, \ldots, \mathbf{x}_{n}$ be subsets of $\left\{X_{1}, \cdots, X_{d}\right\}$ with $r_{1}, \ldots, r_{n}$ elements respectively. If $i_{1}, \ldots, i_{n}$ are integers then

$$
\operatorname{length}\left(H_{i_{1}}\left(\mathbf{x}_{1} ; H_{i_{2}}\left(\mathbf{x}_{2} ; \ldots ; H_{i_{n}}\left(\mathbf{x}_{n} ; M\right) \ldots\right)\right) \geq \prod_{j=1}^{n}\left(\begin{array}{c}
r_{j} \\
i_{j}
\end{array}\right) .\right.
$$

This proposition becomes a tempting conjecture in the absence of multi-grading. However, as the example in the Appendix shows, this is too much to hope for in general though one might speculate on the possibility that with a suitable change of parameters the inequality might survive intact.

A much greater debt is due Evans and Griffith for the techniques of their Theorem 2.4 [ $\mathbf{E G}_{2}$ ] of which extensive use is made in this paper. Lemma 2.1 abstracts the essential elements of the techniques. Finally a great deal of thanks is due Melvin Hochster who made a number valuable remarks instrumental in the development of this paper.

2. The one-variable case. Throughout this section let $R$ be any commutative Noetherian ring with identity. In Lemma 2.1 below and in many other places in this manuscript, we refer to a class $\mathscr{E}$ of modules together with an additive function $\lambda$ taking on values in an ordered abelian group. In most of our applications, $\lambda$ is simply length and, if one wishes, the more general results may be rendered more palatable by taking this view. There are, however, many additive functions other than length for which the inequalities of Theorems 3.3 and 5.2 have applications. Example 3.4 gives such an application where $\lambda$ is defined via Serre's intersection multiplicity.

Of immediate concern is a preliminary version of Theorem 3.3. Three easy lemmas set up the theorem.

LEMMA 2.1. Let $\mathscr{C}$ be a class of $R$-modules which is closed with respect to forming submodules and quotient modules and let $\lambda$ be any additive function defined on $\mathscr{C}$ taking on values in an ordered abelian 
group with $\lambda(C) \geq 0$ for $C \in \mathscr{C}$ (e.g. $\lambda=$ length and $\mathscr{C}$ the class of finite length $R$-modules). Given either of the following (dual) commutative diagrams in $\mathscr{C}$

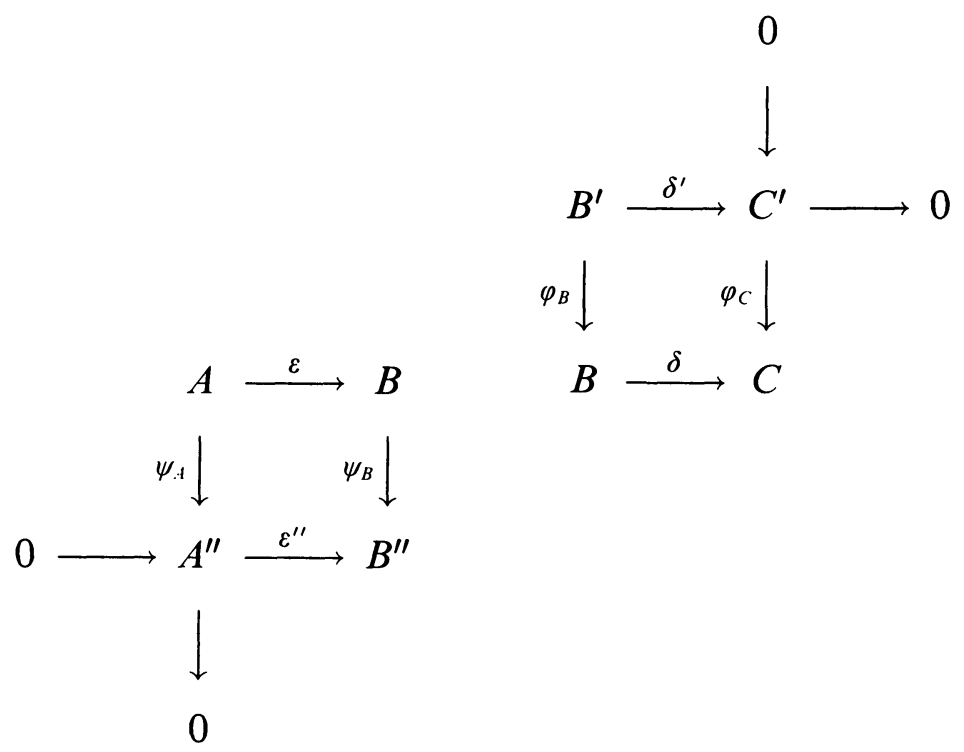

with injections $\varphi_{C}$ and $\varepsilon^{\prime \prime}$ and surjections $\delta^{\prime}$ and $\psi_{A}$ then:
(a) $\lambda(\operatorname{im} \delta) \geq \lambda\left(\operatorname{im} \delta^{\prime}\right)$,
(b) $\lambda(\mathrm{im} \varepsilon) \geq \lambda\left(\mathrm{im} \varepsilon^{\prime \prime}\right)$.

Furthermore, given both diagrams with $\delta \varepsilon=0$ then

(c) $\lambda(B) \geq \lambda\left(\mathrm{im} \varepsilon^{\prime \prime}\right)+\lambda\left(\mathrm{im} \delta^{\prime}\right)$.

Proof. (a) $\operatorname{im} \varphi_{c} \delta^{\prime}=\operatorname{im} \delta \varphi_{B}$ and $\operatorname{im} \delta \supseteq \operatorname{im} \delta \varphi_{B}$ so clearly

$$
\lambda(\operatorname{im} \delta) \geq \lambda\left(\operatorname{im} \varphi_{C} \delta^{\prime}\right) .
$$

But $\varphi_{C}$ is injective and $\delta^{\prime}$ is surjective so

$$
\lambda\left(\operatorname{im} \varphi_{C} \delta^{\prime}\right)=\lambda\left(\operatorname{im} \delta^{\prime}\right) .
$$

Hence $\lambda(\operatorname{im} \delta) \geq \lambda\left(\operatorname{im} \delta^{\prime}\right)$. (b) follows by virtually the same argument. For $(\mathrm{c})$, it is only necessary to observe that $\delta \varepsilon=0 \Rightarrow \lambda(B) \geq \lambda(\mathrm{im} \varepsilon)+$ $\lambda(\operatorname{im} \delta)$ so that the result follows from (a) and (b).

LemMA 2.2. Consider the functorial short exact sequence of $R$ modules:

$$
0 \rightarrow R[X] \otimes_{R} A \stackrel{\sigma}{\rightarrow} R[X] \otimes_{R} A \stackrel{\varepsilon}{\rightarrow} A \rightarrow 0
$$


where

$$
\sigma\left(\sum_{i} X^{i} \otimes a_{i}\right)=\sum_{i} X^{i}(X \otimes 1-1 \otimes X) a_{i},
$$

$\varepsilon(f \otimes a)=f a$ and $A$ is any $R[X]-$ module $\left[\mathbf{E G}_{\mathbf{1}} ;\right.$ Syzygies, $\left.p .244\right]$. The map $\sigma$ is equivalent to multiplication by $X$ if and only if $X A=0$.

Proof. Immediate from the definition of $\sigma$. See [EG $\mathbf{2}$, Lemma 2.3].

Note. The $R[X] \otimes_{R} A$ terms in Lemma 2.2 are $R[X]$-modules via $R[X]$ on the left or $A$ on the right. It is the left-hand structure intended here. I.e. $f(s \otimes a)=f s \otimes a$.

LEMMA 2.3. Let $R \subseteq S$ be rings with $S$ flat over $R$. Let $K$ be an $S$-module and $A$ any $R$-module. Then

$$
\operatorname{Tor}_{i}^{S}\left(K, S \otimes_{R} A\right) \cong \operatorname{Tor}_{i}^{R}(K, A) \quad \forall i \geq 0 .
$$

Proof. This result follows in a straightforward manner from the definition of Tor. Let $F_{\bullet}$ be an $R$-free resolution of $A$. $\operatorname{Tor}_{i}^{R}(K, A)$ is computed by taking the homology of $K \otimes_{R} F_{\text {• }}$. Since $S$ is $R$-flat, $S \otimes_{R} F_{\bullet}$ is a $S$-free resolution of $S \otimes_{R} A$. Thus $\operatorname{Tor}_{i}^{S}\left(K, S \otimes_{R} A\right)$ may be computed by taking the homology of $K \otimes_{S} S \otimes_{R} F_{\bullet}$. However $K \otimes_{S} S \otimes_{R} \mathbf{F}_{\bullet} \cong$ $K \otimes_{R} F_{0}$. so the results are the same.

Definition 2.4. We will say that an $R[X]$-module $M$ admits a highlow decomposition if there are non-zero $R$-modules $M_{h}$ and $M_{l}$ each killed by $X$ such that there are:

(1) An $R[X]$-module injection

$$
0 \rightarrow M_{h} \rightarrow M \quad \text { which splits over } R
$$

and

(2) An $R[X]$-module surjection

$$
M \rightarrow M_{l} \rightarrow 0 \quad \text { which splits over } R .
$$

In the case where $M \neq 0$ is killed by a power of $X$ and is $\mathbb{Z}$-graded over $R$, i.e. $M=\bigoplus_{i \in \mathbb{Z}} M_{i}$, we may choose $M_{l}=M_{i}$ where $i$ is the smallest integer such that $M_{i} \neq 0$ and $M_{h}=M_{j}$ where $j$ is the largest integer such that $M_{j} \neq 0$. 
THEOREM 2.5. Let $K$ be an $R[X]$-module such that $X K=0$. Let $\lambda$ be an additive function as in Lemma 2.1 defined on a class $\mathscr{C}$ of $R[X]$-modules which is closed with respect to taking submodules and quotient modules. Assume also that $\operatorname{Tor}_{i}^{R[X]}(K$, ) sends members of $\mathscr{C}$ to members of $\mathscr{C}$. Let $M \in \mathscr{C}$ be a module which admits a high-low decomposition. Then

$$
\lambda\left(\operatorname{Tor}_{i}^{R[X]}(K, M)\right) \geq \lambda\left(\operatorname{Tor}_{i}^{R}\left(K, M_{l}\right)\right)+\lambda\left(\operatorname{Tor}_{i-1}^{R}\left(K, M_{h}\right)\right) .
$$

Proof. Since the sequence in Lemma 2.2 is functorial, the high-low decomposition yields two commuting diagrams:

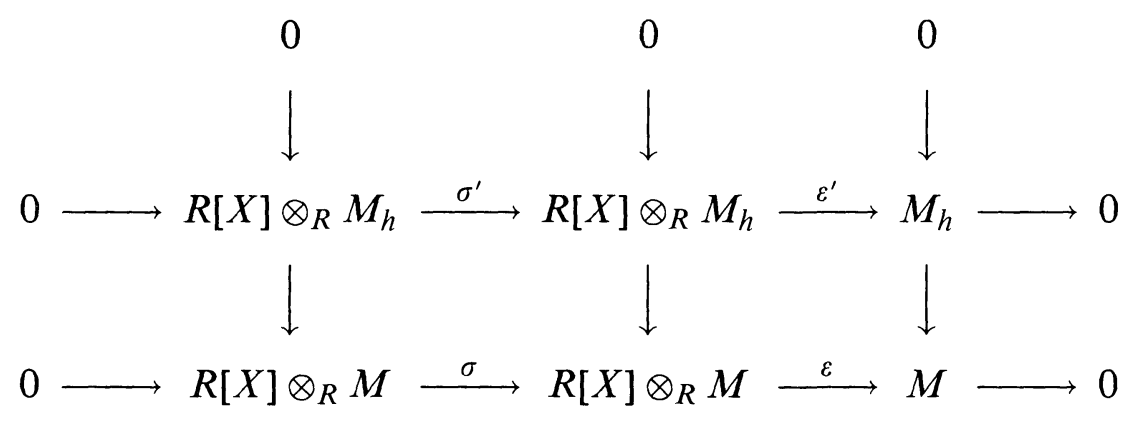

and

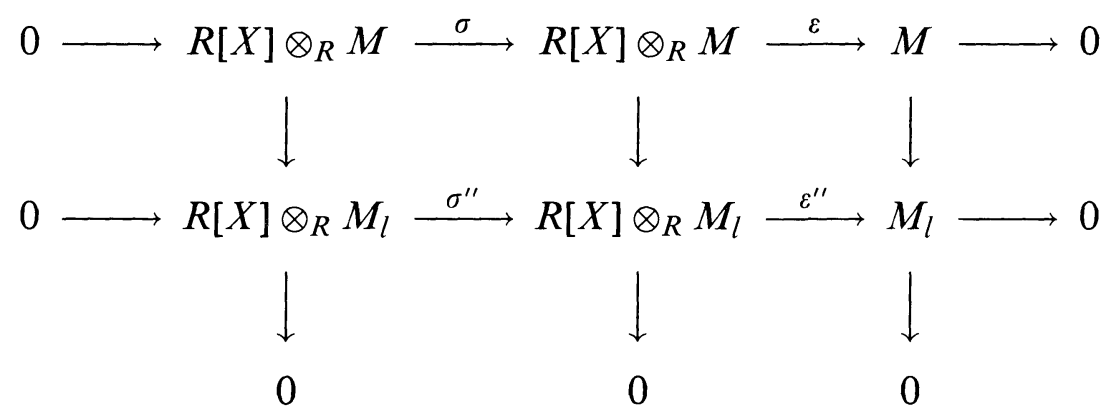

Note that in both diagrams all columns are $R[X]$-exact but the first two columns are $R[X]$-split exact. Thus, applying $\operatorname{Tor}_{\bullet}^{R[X]}(K$,$) and$ making the reduction

$$
\operatorname{Tor}_{i}^{R[X]}\left(K, R[X] \otimes_{R} A\right) \cong \operatorname{Tor}_{i}^{R}(K, A)
$$


from Lemma 2.3 yields:

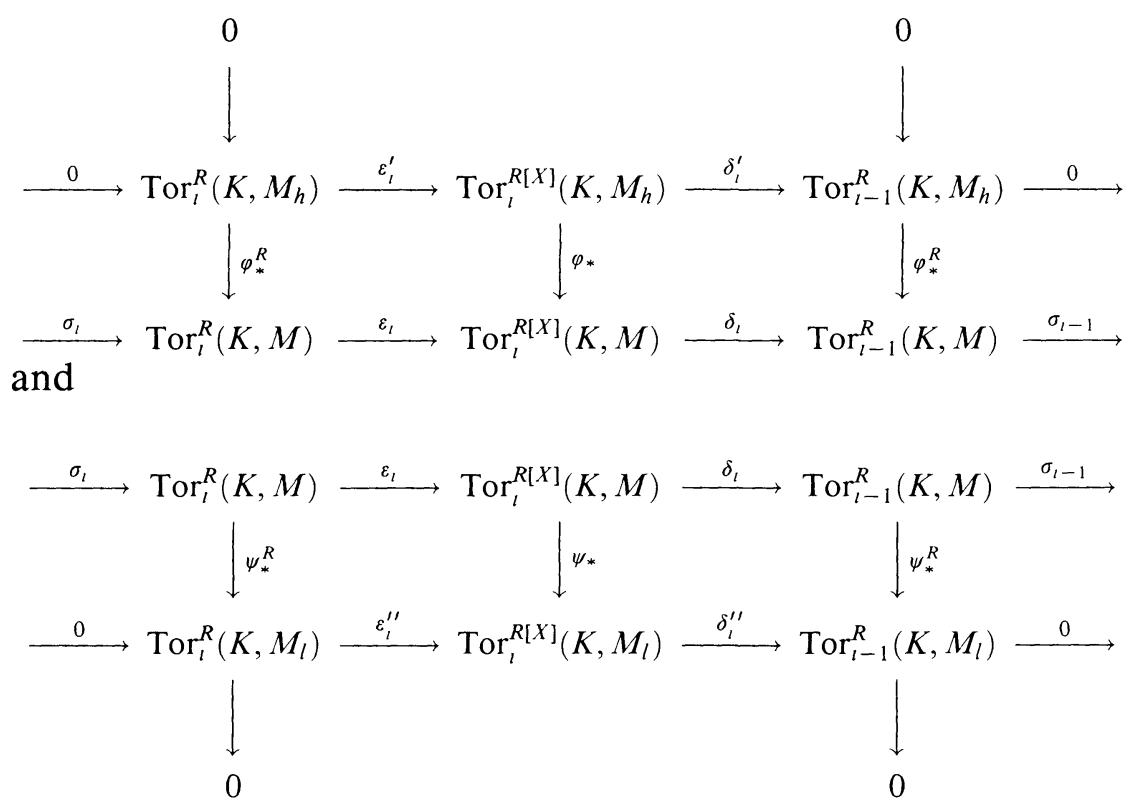

Finally, noting that Lemma 2.2 forces all of the $\sigma_{i}^{\prime}$ and $\sigma_{i}^{\prime \prime}$ to be zero maps, we find ourselves in the context of Lemma 2.1 from which the result is merely the rephrasing of (c).

COROLlaRy 2.6. Let $(R, m, k)$ be an $n$-dimensional regular local ring for which Horrocks' question is known to have an affirmative answer. I.e. assume that for any finite length $R$-module $A, b_{i}(A) \geq\left(\begin{array}{l}n \\ l\end{array}\right)$ where $b_{i}(A)=\operatorname{dim}_{k} \operatorname{Tor}_{i}^{R}(k, A)$ is the ith Betti number of $A$. Then for all $R[X]$-modules $M$ which admit high-low decompositions

$$
\operatorname{dim}_{k} \operatorname{Tor}_{i}^{R[X]}(k, M) \geq\left(\begin{array}{c}
n+1 \\
i
\end{array}\right) .
$$

Proof. This result follows immediately from Theorem 2.5 letting $K=k, \mathscr{C}$ be the class of finite length $R[X]$-modules, $\lambda$ be $\operatorname{dim}_{k}$, which coincides with length on $\mathscr{C}$, and the conbinatorial identity

$$
\left(\begin{array}{c}
n \\
i
\end{array}\right)+\left(\begin{array}{c}
n \\
i-1
\end{array}\right)=\left(\begin{array}{c}
n+1 \\
i
\end{array}\right)
$$

with the convention that $\left(\begin{array}{c}n \\ i\end{array}\right)=0$ if $n<0$ or $n<i$.

REMARK 2.7. The existence of high-low decomposition of Corollary 2.6 imposes definite restrictions on $M$ : There are examples where no 
choice of $M_{h}$ or $M_{l}$ suffices. Since $M$ has finite length, we have

$$
M \supsetneq X M \supsetneq X^{2} M \supsetneq \cdots \supsetneq X^{p} M \supsetneq 0
$$

for some $p>0$. Thus it is natural to try to take $M_{h}=X^{p} M$ and $M_{l}=M / X M$. Unfortunately there remains the problem of showing that the injection and quotient maps split over $R$ which is not generally possible. For example let $k$ be a field and $R=k[Y]$ and let $M=$ $R[X, Y] /\left(X-Y, X^{2}\right)$ which is isomorphic to $k[Y] /\left(Y^{2}\right) . M$ is killed by $X^{2}$ but is not decomposable over $k[Y]$. (See also Remarks 3.8 and 4.5.)

Having gained a modest toehold in the direction of grading, it is natural to generalize the notion of high-low decomposition to modules over $R\left[X_{1}, \ldots, X_{d}\right]$. This is the subject of $\S 3$.

3. The multi-variable case. The direction of generalization to $R\left[X_{1}, \ldots, X_{d}\right]$, where $R$ is Noetherian, is clear in the sense that we expect a result similar to Theorem 2.5 when the module $M$ has a highlow decomposition in some sense compatible with reduction to smaller and smaller polynomial rings over $R$. The idea is simple enough: Pick an $X$ and find $M_{h}$ and $M_{l}$; pick another $X$ and find $\left(M_{h}\right)_{h},\left(M_{h}\right)_{l}$, $\left(M_{l}\right)_{h}$ and $\left(M_{l}\right)_{l}$; etc. The actual implementation of this scheme requires some bookkeeping rules.

For the remainder of this section let $S=R\left[X_{1}, \ldots, X_{d}\right]$.

Definitions 3.1. Let $W_{i}$ be the set of all strings of l's and $h$ 's of length $i$. For example: $W_{2}=\{h h, h l, l h, l l\}$ and $W_{0}=\{\varnothing\}$ where $\varnothing$ denotes the empty string. For $w \in W_{i}$ define $\|w\|=i$, the total length of $w$, and denote the number of $h$ 's that appear in $w$ by $\mathrm{h}(w)$. E.g. $\|h l h h l\|=5$ and $h(h l h h l)=3$.

We will say that an $S$-module $M$ admits a high-low decomposition with respect to $X_{1}, \ldots, X_{d}$ if there is a family of $S$-modules indexed by $W=\bigcup_{0}^{d} W_{i}$ satisfying:

(i) $M_{\varnothing}=M$,

(ii) $X_{i} M_{w}=0$ whenever $i+\|w\|>d$,

(iii) For all $w \in W_{d-i}, 1 \leq i \leq d$ there are,

$$
S \text {-module injections } 0 \rightarrow M_{w h} \rightarrow M_{w}
$$

and

$$
S \text {-module injections } M_{w} \rightarrow M_{w l} \rightarrow 0
$$

which are split over $R\left[X_{1}, \ldots, X_{i-1}\right]$.

REMARK 3.2. Of particular interest are $\mathbb{Z}^{d}$-graded modules over $R\left[X_{1}, \ldots, X_{d}\right]$ which are killed by a power of $\left(X_{1}, \ldots, X_{d}\right)$, since they 
always have high-low decompositions with respect to any arrangement of the $X$ 's. Thus if $M=\bigoplus\left\{M_{(n)}:(n) \in \mathbb{Z}^{d}\right\}$ is a non-zero $\mathbb{Z}^{d}$-graded module killed by a power of $\left(X_{1}, \ldots, X_{d}\right)$ over $R\left[X_{1}, \ldots, X_{d}\right]$ and $\nu$ is an integer, define $M_{\nu}=\bigoplus\left\{M_{(n)}: n_{d}=\nu\right\}$. Then set $M_{l}=M_{\nu}$ where $\nu$ is the smallest integer such that $M_{\nu} \neq 0$ and $M_{l}=M_{\nu}$ where $\nu$ is the largest integer such that $M_{\nu} \neq 0$. This takes care of a highlow decomposition with respect to the last variable $X_{d}$. Now one can use $\|w\|$ to recursively define the $M_{w}$ by letting $M_{w l}=\left(M_{w}\right)_{l}$ and $M_{w l}=\left(M_{w}\right)_{h}$ where the $l$ and $h$ operations are taken with respect to $X_{d-\|w\|}$. One easily checks that conditions (i), (ii) and (iii) are satisfied, keeping in mind that adding an $h$ identifies a submodule while adding an $l$ creates a quotient module.

We are now in a position to state the main result. Recall that $S=$ $R\left[X_{1}, \ldots, X_{d}\right]$ with $R$ Noetherian.

THEOREM 3.3. Let $\mathscr{C}$ be a class of $S$-modules and $\lambda$ an additive function defined on $\mathscr{C}$ as in Lemma 2.1. Let $K$ be an $S$-module killed by $S_{+}=\left(X_{1}, \ldots, X_{d}\right)$ and assume $\operatorname{Tor}_{i}^{S}(K, A) \in \mathscr{C}$ whenever $A \in \mathscr{C}$. Let $M \in \mathscr{C}$ be a module which admits a high-low decomposition with respect to $X_{1}, \ldots, X_{d}$. Then

$$
\lambda\left(\operatorname{Tor}_{i}^{S}(K, M)\right) \geq \sum_{w \in W_{d}} \lambda\left(\operatorname{Tor}_{i-\mathrm{h}(w)}^{R}\left(K, M_{w}\right)\right) .
$$

Proof. Theorem 3.3 follows by repeated application of Theorem 2.5. The only fine point to note is that the index of the Tor's inside the sum drops according to the number of $h$ 's in $w$. I.e. adding an $h$ causes an index shift as is explicit in Theorem 2.5. As always, $\operatorname{Tor}_{i}\left(K, M_{w}\right)=0$ for $i<0$.

EXAMPLE 3.4. Let $(R, m)$ be a regular local ring containing a field, and let $I$ and $J$ be ideals in $R$ such that $I+J$ is primary to $m$. Let $\mathscr{C}$ be the multi-graded $S$-modules killed by a power of $J+\left(X_{1}, \ldots, X_{d}\right)$ and let $N$ be a (fixed) finitely generated $S$-module killed by a power of $I$. Then for $M \in \mathscr{C}$ let

$$
\lambda(M)=\chi_{R}(M, N)=\sum_{i=0}^{\infty}(-1)^{i} \text { length }\left(\operatorname{Tor}_{i}^{R}(M, N)\right) .
$$

$\lambda$ is nonnegative and additive in $M$. Further, if

$$
\operatorname{dim}_{R} M+\operatorname{dim}_{R} N=\operatorname{dim} R,
$$


then Serre's Theorem gives $\chi_{R}(M, N)>0$. That is, $\chi_{R}$ is positive on a subclass of $\mathscr{C}$. Thus Theorem 3.3 can be applied to produce

$$
\chi_{R}\left(\operatorname{Tor}_{i}^{S}(K, M), N\right) \geq \sum_{w \in W_{d}} \chi_{R}\left(\operatorname{Tor}_{i-\mathrm{h}(w)}^{R}\left(K, M_{w}\right), N\right) .
$$

Note that if $I=(0), J=m$, and $N=R$, then $\chi_{R}(M, N)=$ length $M$ and we are back to the original Horrocks' question.

REMARK 3.5. Notice that if we view $R$ as an $S$-module via $R \cong$ $S /\left(X_{1}, \ldots, X_{d}\right)$, then we may take $K=R$ and Theorem 3.3 becomes

$$
\lambda\left(\operatorname{Tor}_{i}^{S}(R, M)\right) \geq \sum_{\mathrm{h}(w)=i} \lambda\left(\operatorname{Tor}_{0}^{R}\left(R, M_{w}\right)\right)=\sum_{\mathrm{h}(w)=i} \lambda\left(M_{w}\right)
$$

which may provide quite strong lower bounds for $\lambda\left(\operatorname{Tor}_{i}^{S}(R, M)\right)$ depending on the high-low decomposition involved. At the very least it guarantees that $\lambda\left(\operatorname{Tor}_{i}^{S}(R, M)\right) \geq\left(\begin{array}{l}d \\ i\end{array}\right)$ when $\lambda$ takes on integer values and is positive on non-zero modules. In $\S \S 4$ and 5 we will be very interested in the fact that this particular form of Theorem 3.3 is actually a statement about Koszul homology:

$$
\lambda\left(H_{i}\left(X_{1}, \ldots, X_{d} ; M\right)\right) \geq \sum_{\mathrm{h}(w)=i} \lambda\left(M_{w}\right) .
$$

Now if $R$ is local and $K$ is the residue class field of $R$, we arrive at a significant addition to the results known about Horrocks' question.

COROLlary 3.6. Let $(R, m, k)$ be an $n$-dimensional regular local ring for which Horrocks' question is known to have an affirmative answer. Then the question has an affirmative answer for $R\left[X_{1}, \ldots, X_{d}\right]-$ modules $M$ which admit high-low decompositions with respect to $X_{1}, \ldots, X_{d}$ and are killed by a power of the maximal ideal $m S+S_{+}$:

$$
\operatorname{dim}_{k} \operatorname{Tor}_{i}^{S}(k, M) \geq\left(\begin{array}{c}
n+d \\
i
\end{array}\right)
$$

In particular, this settles Horrocks' question for finite length modules that are $\mathbb{Z}^{d}$-graded over $R\left[X_{1}, \ldots, X_{d}\right]$ where $\operatorname{dim} R \leq 4$.

Proof. In this situation Theorem 3.3 reads

$$
\operatorname{dim}_{k}\left(\operatorname{Tor}_{i}^{S}(k, M)\right) \geq \sum_{w \in W_{d}} \operatorname{dim}_{k}\left(\operatorname{Tor}_{i-\mathrm{h}(w)}^{R}\left(k, M_{w}\right)\right) .
$$


By hypothesis each of the terms on the right is at least $\left(\begin{array}{c}n \\ i-\mathrm{h}(w)\end{array}\right)$. For given $j$ there are $\left(\begin{array}{l}d \\ j\end{array}\right) w$ 's in $W_{d}$ with $\mathrm{h}(w)=j$, so that

$$
\begin{aligned}
\operatorname{dim}_{k}\left(\operatorname{Tor}_{i}^{S}(k, M)\right) & \geq \sum_{w \in W_{d}} \operatorname{dim}_{k}\left(\operatorname{Tor}_{i-\mathrm{h}(w)}^{R}\left(k, M_{w}\right)\right) \\
& \geq \sum_{j=0}^{i}\left(\begin{array}{c}
n \\
i-j
\end{array}\right)\left(\begin{array}{c}
d \\
j
\end{array}\right)=\left(\begin{array}{c}
n+d \\
i
\end{array}\right) .
\end{aligned}
$$

REMARK 3.7. Notice that the proof of Corollary 3.6 actually contains the stronger intermediate inequality

$$
\operatorname{dim}_{k}\left(\operatorname{Tor}_{i}^{S}(k, M)\right) \geq \sum_{w \in W_{d}} \operatorname{dim}_{k}\left(\operatorname{Tor}_{i-\mathrm{h}(w)}^{R}\left(k, M_{w}\right)\right)
$$

which gives a lower bound for $\operatorname{dim}_{k} \operatorname{Tor}_{i}^{S}(k, M)$ in terms of lengths associated with the $R$-submodules $M_{w}$.

REMARK 3.8. The example of Remark 2.7 shows that for a fixed choice of generators $X_{1}, \ldots, X_{d}$ of a polynomial ring $S$ over $k$, a module $M$ killed by a power of $\left(X_{1}, \ldots, X_{d}\right)$ may have no high-low decomposition. However, it is conceivable that a module might have a high-low decomposition with respect to a different choice of generators.

4. Koszul homology. Let $R$ be some base ring, $S=R\left[X_{1}, \ldots, X_{d}\right]$ and $M$ an $S$-module. The Koszul homology of $M, H_{\bullet}\left(X_{1}, \ldots, X_{d} ; M\right)$, can be defined as $\left.\operatorname{Tor}_{\bullet} S_{(} S /\left(X_{1}, \ldots, X_{d}\right), M\right) \cong \operatorname{Tor}_{\bullet} S_{(R, M)}$. Thus Theorem 3.3 and Corollary 3.6 apply to these homology groups as well. However the techniques involved in the proof of Theorem 3.3-or, more precisely, its prototype, Theorem 2.5-suggest that quite specific information might be available about the ranks of certain maps between Koszul homology groups (modules). The nature of these maps and some results concerning them are contained in Definition 4.3 and Theorem 4.4.

Let $S$ be a Noetherian ring with $\mathbf{t}=t_{1}, \ldots, t_{d}$ elements of some distinguished maximal ideal $m$. Throughout most of this section we will want to view the Koszul complex $K_{\bullet}(\mathbf{t} ; S)$ as the exterior algebra $\bigwedge^{\bullet} S^{d}$. Thus $K_{i}(\mathbf{t} ; S)$ will be thought of as the free $S$-module on the $\left(\begin{array}{c}d \\ i\end{array}\right)$ generators $u_{\alpha_{1}} \wedge \cdots \wedge u_{\alpha_{i}}, 1 \leq \alpha_{1}<\cdots<\alpha_{i} \leq d$, where $u_{\alpha}$ is a fixed 
generator of the $\alpha$ th component of $S^{d}$, with differential maps

$$
\begin{gathered}
\bigwedge_{i}^{i} S^{d} \stackrel{d_{l}}{\rightarrow} \bigwedge^{i-1} S^{d} \\
u_{\alpha_{1}} \wedge \cdots \wedge u_{\alpha_{1}} \mapsto \sum_{\nu=1}^{i}(-1)^{\nu+1} t_{\alpha_{\nu}} u_{\alpha_{1}} \wedge \cdots \wedge \widehat{u}_{\alpha_{\nu}} \wedge \cdots \wedge u_{\alpha_{l}} .
\end{gathered}
$$

$K$. becomes an associative, skew-commutative, graded differential, free $S$-algebra on the $u_{\alpha}$ under the $\wedge$-product in the usual way. Finally, if $M$ is an $S$-module, the Koszul complex of $M, K_{\bullet}(\mathbf{t} ; M)$ is defined as $K_{\bullet}(\mathbf{t} ; S) \otimes_{R} M$ and the Koszul homology $H_{i}(\mathbf{t} ; M)$ as $H_{i}\left(K_{\bullet}(\mathbf{t} ; M)\right)$.

REMARK 4.1. There is a short exact sequence of complexes

$$
\begin{aligned}
0 \rightarrow K_{i}\left(t_{1}, \ldots, t_{r-1} ; S\right) \stackrel{i}{\rightarrow} & K_{i}\left(t_{1}, \ldots, t_{r} ; S\right) \\
& \stackrel{q}{\rightarrow} K_{i}\left(t_{1}, \ldots, t_{r} ; S\right) / K_{i}\left(t_{1}, \ldots, t_{r-1} ; S\right) \rightarrow 0
\end{aligned}
$$

where $i$ is the obvious inclusion map (sending $u_{\alpha}$ to $u_{\alpha}$ ) and $q$ is the canonical quotient map. (Note: We are appealing to the free $S$-algebra structure of $K_{\bullet}(\mathbf{t} ; S)$ when defining these maps in this fashion.) This of course gives rise to a long exact sequence on homology. We wish, however, to make a reduction on the last term, which is isomorphic to $K_{i-1}\left(t_{1}, \ldots, t_{r-1} ; S\right)$. To see this we need only observe that any surviving generators in

$$
K_{i}\left(t_{1}, \ldots, t_{r} ; S\right) / K_{i}\left(t_{1}, \ldots, t_{r-1} ; S\right)
$$

must involve $u_{r}$ so that with some abuse of notation we might choose to write

$$
K_{i-1}\left(t_{1}, \ldots, t_{r-1} ; S\right) \wedge u_{r}
$$

for

$$
K_{i}\left(t_{1}, \ldots, t_{r} ; S\right) / K_{i}\left(t_{1},, \ldots, t_{r-1} ; S\right) .
$$

This notation is justified since it is compatible with the differential maps:

$$
\begin{aligned}
& d_{i}\left(u_{\alpha_{1}} \wedge \cdots \wedge u_{\alpha_{r-1}} \wedge u_{r}\right) \\
& \quad=d_{i-1}\left(u_{\alpha_{1}} \wedge \cdots \wedge u_{\alpha_{r-1}}\right) \wedge u_{r}+(-1)^{r+1} t_{r} u_{\alpha_{1}} \wedge \cdots \wedge u_{\alpha_{r-1}} \\
& \quad=d_{i-1}\left(u_{\alpha_{1}} \wedge \cdots \wedge u_{\alpha_{r-1}}\right) \wedge u_{r} .
\end{aligned}
$$

Thus the sequence becomes

$$
\begin{aligned}
0 \rightarrow K_{i}\left(t_{1}, \ldots, t_{r-1} ; S\right) \stackrel{i}{\rightarrow} K_{i}\left(t_{1}, \ldots, t_{r} ; S\right) & \\
& \stackrel{q}{\rightarrow} K_{i-1}\left(t_{1}, \ldots, t_{r-1} ; S\right) \wedge u_{r} \rightarrow 0
\end{aligned}
$$


Tensoring with $M$ and taking homology produces a long exact sequence on Koszul homology. However, for the moment we will only be interested in the segment

$$
H_{i}\left(t_{1}, \ldots, t_{r-1} ; M\right) \stackrel{i_{*}}{\rightarrow} H_{i}\left(t_{1}, \ldots, t_{r} ; M\right) \stackrel{q_{*}}{\rightarrow} H_{i-1}\left(t_{1}, \ldots, t_{r-1} ; M\right)
$$

with $q_{*} i_{*}=0$. We may also uncouple these maps to generate another kind of map:

$$
H_{i}\left(t_{1}, \ldots, t_{r} ; M\right) \stackrel{q_{*}}{\rightarrow} H_{i-1}\left(t_{1}, \ldots, t_{r-1} ; M\right) \stackrel{i_{*}}{\rightarrow} H_{i-1}\left(t_{1}, \ldots, t_{r-1}, t_{s} ; M\right)
$$

with $s>r$. It is maps of this latter type that can be squeezed for considerably more information.

REMARK 4.2. The notions of dropping and adding $t$ 's with these quotient and inclusion maps generalizes in a natural enough way. Let $\mathbf{x}=x_{1}, \ldots, x_{r}, \mathbf{y}=y_{i}, \cdots, y_{s}$ and $\mathbf{z}=z_{1}, \ldots, z_{t}$ be sequences of members of $m$ with corresponding generators $u_{1}, \ldots, u_{r}, v_{1}, \ldots, v_{s}$, $w_{1}, \ldots, w_{t}$ in $K_{1}(\mathbf{x} ; S), K_{1}(\mathbf{y} ; S)$ and $K_{1}(\mathbf{z} ; S)$ respectively. The inclusion and quotient maps of Remark 4.1 become:

$$
\begin{gathered}
K_{i}(\mathbf{x} ; S) \stackrel{i}{\rightarrow} K_{i}(\mathbf{x}, \mathbf{z} ; S), \\
u_{\alpha_{1}} \wedge \cdots \wedge u_{\alpha_{t}} \mapsto u_{\alpha_{1}} \wedge \cdots \wedge u_{\alpha_{i}}
\end{gathered}
$$

and

$$
\begin{gathered}
K_{i}(\mathbf{x}, \mathbf{y}, \mathbf{z} ; S) \stackrel{q}{\rightarrow} K_{i-s}(\mathbf{x}, \mathbf{z} ; S) \wedge v_{1} \wedge \cdots \wedge v_{s}, \\
u_{\alpha_{1}} \wedge \cdots \wedge u_{\alpha_{r^{\prime}}} \wedge v_{\beta_{1}} \wedge \cdots \wedge v_{\beta_{s^{\prime}}} w_{\gamma_{1}} \wedge \cdots \wedge w_{\gamma_{t^{\prime}}} \\
\mapsto\left\{\begin{array}{l}
0, \quad \text { if } s^{\prime}<s \text { (not all } v \text { s occur) } \\
\left(u_{\alpha_{1}} \wedge \cdots \wedge u_{\alpha_{r^{\prime}}} \wedge w_{\gamma_{1}} \wedge \cdots \wedge w_{\gamma^{\prime}}\right) \wedge v_{1} \wedge \cdots \wedge v_{s} \quad \text { otherwise. }
\end{array}\right.
\end{gathered}
$$

The inclusion map is quite straightforward and presents no intrinsic difficulties. The quotient map however, as described, is potentially much more complicated in that it does not agree with the usual conventions for interchanging the order of generators in a skew commutative algebra. This will turn out to be a harmless simplification.

The maps induced on homology by the above quotient and inclusion maps are of a type tractable to the techniques of Theorem 2.5. To this end we make an ad hoc definition.

Definition 4.3. Let $\mathbf{x}, \mathbf{y}$ and $\mathbf{z}$ be disjoint sequences in $m$ as in Remark 4.2. A map

$$
\eta_{i, \mathbf{x}, \mathbf{y}, \mathbf{z}}: H_{i+s}(\mathbf{x}, \mathbf{y} ; M) \rightarrow H_{i}(\mathbf{x} ; M) \rightarrow H_{i}(\mathbf{x}, \mathbf{z} ; M)
$$


will be called an $\eta$-map or of $\eta$-type if it is induced by a composition of quotient followed by inclusion of the form described in Remark 4.2. $\eta_{i, \mathbf{x}, \mathbf{y}, \mathbf{z}}$ will be denoted by $\eta_{i}$ or just $\eta$ when the context is clear.

As in $\S 3$ our concern will be with a polynomial ring $S=$ $R\left[X_{1}, \ldots, X_{d}\right]$ over some base ring $R$.

THEOREM 4.4. Let $S=R\left[X_{1}, \ldots, X_{d}\right]$ be the polynomial ring in $d$ variables over a ring $R$. Let $\mathscr{C}$ be a class of $S$-modules and $\lambda$ an integervalued additive function defined on $\mathscr{C}$ as in Lemma 2.1 and assume $\operatorname{Tor}_{i}^{S}(R, A) \in \mathscr{C}$ whenever $A \in \mathscr{C}$. Also assume $\lambda(A)=0 \Rightarrow A=0$. Let $M \in \mathscr{C}$ be a module which admits a high-low decomposition with respect to $X_{1}, \ldots, X_{d}$ and let

$$
\mathbf{x}, \mathbf{y}, \mathbf{z}=x_{1}, \ldots, x_{r}, y_{1}, \ldots, y_{s}, z_{1}, \ldots, z_{t}
$$

be an ordered partition of the $X$ 's with corresponding $\eta$-map

$$
\eta_{i, \mathbf{x}, \mathbf{y}, \mathbf{z}}: H_{i+s}(\mathbf{x}, \mathbf{y} ; M) \rightarrow H_{i}(\mathbf{x}, \mathbf{z} ; M) .
$$

Then $\lambda\left(\operatorname{im} \eta_{i, \mathbf{x}, \mathbf{y}, \mathbf{z}}\right) \geq\left(\begin{array}{l}r \\ i\end{array}\right)$

Proof. We proceed by induction on $r$. Suppose $r=0$. The task at hand is to show that

$$
\begin{gathered}
\lambda(\operatorname{im} \eta \varnothing, y, z) \geq\left(\begin{array}{l}
0 \\
i
\end{array}\right) \text { where } \\
\eta_{\varnothing, y, z}: H_{i+s}(\mathbf{y} ; M) \rightarrow H_{i}(\varnothing ; M) \rightarrow H_{i}(\mathbf{z} ; M) .
\end{gathered}
$$

For $i \neq 0$ this is trivially true. Thus we are left with the case

$$
\eta: H_{s}(\mathbf{y} ; M) \rightarrow H_{0}(\varnothing ; M) \rightarrow H_{0}(\mathbf{z} ; M)
$$

and the problem of showing this map to be non-zero. $\eta$ is induced by the maps

$$
K_{s}(\mathbf{y} ; M) \stackrel{q}{\rightarrow} K_{0}(\varnothing ; M) \stackrel{i}{\rightarrow} K_{0}(\mathbf{z} ; M) .
$$

We observe that $K_{0}(\varnothing ; M)$ is, in fact, $M$ and $q$ sends $m\left(v_{1} \wedge \cdots \wedge v_{s}\right)$ to $(m) \wedge v_{1} \wedge \cdots \wedge v_{s}$ and thence to $(m)$ by $i$. Passing to homology we get

$$
\eta: \operatorname{Ann}_{M}\left(y_{1}, \ldots, y_{s}\right) \rightarrow M \rightarrow M /\left(z_{1}, \ldots, z_{t}\right) M .
$$

I.e. $\eta$ is just the inclusion followed by the quotient, which reduces the problem to showing that

$$
\operatorname{Ann}_{M}\left(y_{1}, \ldots, y_{s}\right) \nsubseteq\left(z_{1}, \ldots, z_{t}\right) M .
$$


It is the high-low decomposition that comes to our rescue in this instance and in a way not at all associated with the commutative diagrams of Theorems 2.5. If we were to restrict ourselves to the monomially graded case, the following argument would be an easy exercise. However the generality of a high-low decomposition demands a bit more care.

Let $w=l \ldots l$ ( $t l$ 's) and $v=h \ldots h$ ( $s h$ 's). The high-low decomposition yields an $S$-module surjection

$$
q: M \rightarrow M_{w} \rightarrow 0
$$

which is split over $R\left[y_{1}, \ldots, y_{s}\right]$ by $\sigma: M_{w} \rightarrow M$ say. Similarly we have an $S$-module injection

$$
0 \rightarrow M_{w v} \rightarrow M_{w}
$$

so that $M_{w v}$ can be considered an actual submodule of $M_{w}$ over $S$ as well as over $R\left[y_{1}, \ldots, y_{s}\right]$. Thus $\sigma\left(M_{w v}\right) \subseteq M$ is non-zero and is killed by the $y$ 's. I.e. $\sigma\left(M_{w v}\right) \subseteq \operatorname{Ann}_{M}\left(y_{1}, \ldots, y_{s}\right)$. We have

$$
M=\sigma\left(M_{w}\right) \oplus \operatorname{ker} q
$$

as $R\left[y_{1}, \ldots, y_{s}\right]$-modules and clearly $\left(z_{1}, \ldots, z_{t}\right) M \subseteq \operatorname{ker} q$ since $q$ is an $S$-module homomorphism. Thus

$$
\sigma\left(M_{w}\right) \cap\left(z_{1}, \ldots, z_{t}\right) M=0
$$

so that

$$
\sigma\left(M_{w v}\right) \subseteq \sigma\left(M_{w}\right) \subseteq \operatorname{Ann}_{M}\left(y_{1}, \ldots, y_{s}\right) \nsubseteq\left(z_{1}, \ldots, z_{t}\right) M
$$

and consequently $\eta$ is not the zero map.

The inductive step, though non-trivial, is mostly a matter of setting up an argument of the same general form as that used in Lemma 2.1. We assume that for all $i \leq d$, and for any $\eta$-type map $\eta_{i, \mathbf{x}, \mathbf{y}, \mathbf{z}}$ where $\mathbf{x}$ has $r-1$ or fewer elements,

$$
\lambda\left(\operatorname{im} \eta_{i, \mathbf{x}, \mathbf{y}, \mathbf{z}}\right) \geq\left(\begin{array}{c}
r-1 \\
i
\end{array}\right) .
$$

Consider the following diagram:

$$
\begin{aligned}
& H_{i+s}\left(x_{1}, \ldots, x_{r-1}, \mathbf{y} ; M\right) \stackrel{\eta^{\prime}}{\longrightarrow} H_{i}\left(x_{1}, \ldots, x_{r-1}, \mathbf{z} ; M\right) \\
& \downarrow i_{*} \quad \downarrow i_{*}^{\prime} \\
& H_{i+s}\left(x_{1}, \ldots, x_{r-1}, x_{r}, \mathbf{y} ; M\right) \stackrel{\eta}{\longrightarrow} H_{i}\left(x_{1}, \ldots, x_{r-1}, x_{r}, \mathbf{z} ; M\right) \\
& \downarrow q_{*} \quad \downarrow q_{*}^{\prime} \\
& H_{i-1+s}\left(x_{1}, \ldots, x_{r-1}, \mathbf{y} ; M\right) \stackrel{\eta^{\prime \prime}}{\longrightarrow} H_{i-1}\left(x_{1}, \ldots, x_{r-1}, \mathbf{z} ; M\right)
\end{aligned}
$$


where $i_{*}, q_{*}, i_{*}^{\prime}$ and $q_{*}^{\prime}$ are the maps described in Remarks 4.1 and 4.2. The $\eta$ 's are $\eta$-type maps with $\eta^{\prime}$ and $\eta^{\prime \prime}$ satisfying the inductive hypothesis on $x_{1}, \ldots, x_{r-1}$. Observe that both $\eta^{\prime \prime} q_{*}$ and $i_{*}^{\prime} \eta^{\prime}$ are $\eta$ type maps satisfying the inductive hypothesis as well. If $N$ is any submodule of $H_{i}\left(x_{1}, \ldots, x_{r-1}, x_{r}, \mathbf{z} ; M\right)$ then

$$
\lambda(N) \geq \lambda\left(\operatorname{im} i_{*}^{\prime} \cap N\right)+\lambda\left(q_{*}^{\prime}(N)\right)
$$

since $q_{*}^{\prime} i_{*}^{\prime}=0$. Since $i_{*}^{\prime} \supseteq \operatorname{im} i_{*}^{\prime} \eta^{\prime}$,

$$
\lambda(N) \geq \lambda\left(\operatorname{im} i_{*}^{\prime} \eta^{\prime} \cap N\right)+\lambda\left(q_{*}^{\prime}(N)\right) .
$$

Thus, if $N=\operatorname{im} \eta$ we have

$$
\lambda(\operatorname{im} \eta) \geq \lambda\left(\operatorname{im} i_{*}^{\prime} \eta^{\prime} \cap \operatorname{im} \eta\right)+\lambda\left(\operatorname{im} q_{*}^{\prime} \eta\right) .
$$

If the squares commute we will then have

$$
\operatorname{im} i_{*}^{\prime} \eta^{\prime} \cap \operatorname{im} \eta=\operatorname{im} i_{*}^{\prime} \eta^{\prime} \text { and } \operatorname{im} q_{*}^{\prime} \eta=\operatorname{im} \eta^{\prime \prime} q_{*}^{\prime}
$$

which, as noted, are $\eta$-type maps satisfying the inductive hypothesis so that

as desired.

$$
\lambda(\operatorname{im} \eta) \geq\left(\begin{array}{c}
r-1 \\
i
\end{array}\right)+\left(\begin{array}{l}
r-1 \\
i-1
\end{array}\right)=\left(\begin{array}{l}
r \\
i
\end{array}\right)
$$

As it turns out, both squares commute at the complex level though checking this can be frustrating if one picks inconvenient conventions. The view presented in Remark 4.2 facilitates this computation which one may verify with the diagrams:

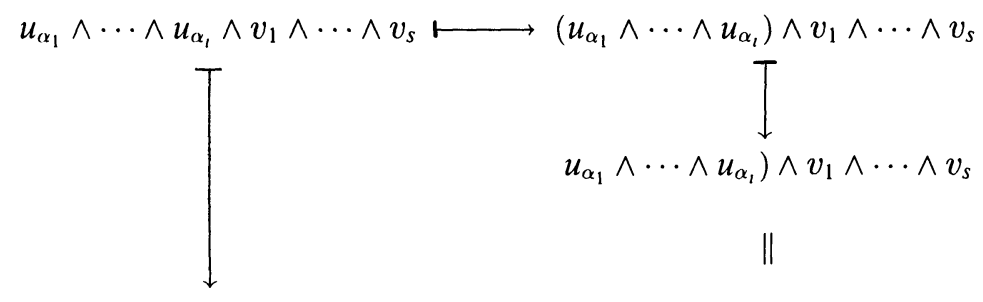

$$
u_{\alpha_{1}} \wedge \cdots \wedge u_{\alpha_{1}} \wedge v_{1} \wedge \cdots \wedge v_{s} \longmapsto\left(u_{\alpha_{1}} \wedge \cdots \wedge u_{\alpha_{l}}\right) \wedge v_{1} \wedge \cdots \wedge v_{s}
$$

and

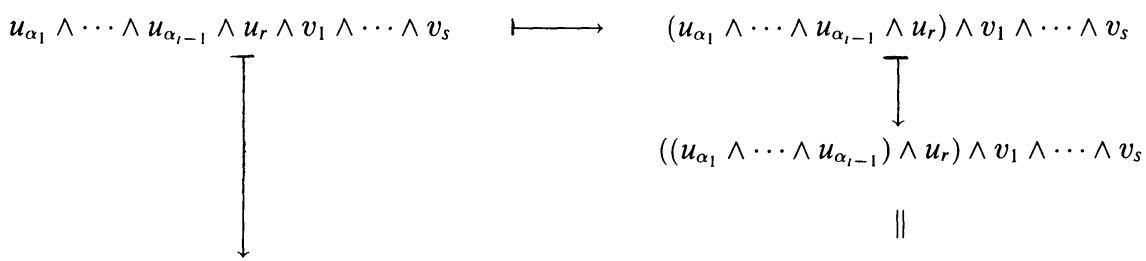

$(-1)^{s}\left(u_{\alpha_{1}} \wedge \cdots \wedge u_{\alpha_{t-1}} \wedge v_{1} \wedge \cdots \wedge v_{s}\right) \wedge u_{r} \longmapsto(-1)^{s}\left(\left(u_{\alpha_{1}} \wedge \cdots \wedge u_{\alpha_{t-1}}\right) \wedge v_{1} \wedge \cdots \wedge v_{s}\right) \wedge u_{r}$ 
REMARK 4.5. The problem of showing

$$
\operatorname{Ann}_{M}\left(y_{1}, \ldots, y_{s}\right) \nsubseteq\left(z_{1}, \ldots, z_{t}\right) M
$$

seems, in general, to be a difficult question since the containment may hold even in very nice situations. The example of Remark 2.7 serves once more to illustrate this point:

$$
R=K[X, Y] \text { and } M=k[X, Y] /\left(X-Y, X^{2}\right) \cong k+k X .
$$

We have

$$
\operatorname{Ann}_{M} X=k X \quad \text { and } \quad Y M=k Y=k X .
$$

REMARK 4.6. Note that if $s=t=0$ then Theorem 4.4 reduces to another solution to Horrocks' question for modules with high-low decompositions.

5. Iterated Koszul homology. A further useful property of high-low decompositions is that "unused portions" are inherited by Koszul homology. This gives rise to a result (Theorem 5.2) on iterated Koszul homology which, despite its formidable appearance, is remarkably easy to prove from our point of view. Furthermore, dropping highlow decomposition from the hypothesis yields a new conjecture which subsumes Horrocks' question. Unfortunately this "conjecture" goes a little too far as demonstrated by the example in the appendix due to M. Hochster and J. McLaughlin. However, as remarked in the introduction, we may still entertain the possibility that there is a reasonable conjecture here with respect to "some" set of parameters.

We proceed with the relative persistence of high-low decompositions through Koszul homology.

LEMMA 5.1. Let $S=R\left[X_{1}, \ldots, X_{r}, Y_{1}, \ldots, Y_{S}\right]$ with $R$ any Noetherian base ring and let $M$ be a finitely generated $S$-module killed by a power of the irrelevant ideal $S_{+}$with associated nonzero $S$-modules $M_{w}$ forming a high-low decomposition with respect to $\mathbf{X}, \mathbf{Y}=X_{1}, \ldots, X_{r}$, $Y_{1}, \ldots, Y_{s}$ as in Definition 3.1. Then the $R\left[Y_{1}, \ldots, Y_{s}\right]$-modules $H_{j}(\mathbf{X} ; M), 0 \leq j \leq r$, have high-lower decompositions with respect to $Y_{1}, \ldots, Y_{s}$ defined by

$$
\left(H_{j}(\mathbf{X} ; M)\right)_{v}=H_{j}\left(\mathbf{X} ; M_{v}\right) \quad \text { for } v \in \bigcup_{0}^{s} W_{i} .
$$

Proof. Before checking the conditions of Definition 3.1, we first verify that the modules proposed for a high-low decomposition of 
$H_{j}(\mathbf{X} ; M)$ are, in fact, nonzero. I.e., we need to show that for $0 \leq j \leq r$ and for $v \in \bigcup_{0}^{s} W_{i}, H_{j}\left(\mathbf{X} ; M_{v}\right) \neq 0$. This follows from the rigidity of Koszul homology, the fact that $M_{v} \neq 0$ and the observation that

$$
H_{r}\left(\mathbf{X} ; M_{v}\right)=\operatorname{Ann}_{M_{v}}(\mathbf{X}) \neq 0 .
$$

Condition (i) of Definition 3.1 is satisfied "by definition" and condition (ii) follows from the fact that

$$
\operatorname{Ann}_{S} \operatorname{Tor}_{j}^{S}(A, B) \supseteq \operatorname{Ann}_{S} A+\operatorname{Ann}_{S} B .
$$

For all $v \in W_{s-\sigma}, 1 \leq \sigma \leq s$, the high-low decomposition of $M$ with respect to $Y$ gives an $R\left[\mathrm{X}, Y_{1}, \ldots, Y_{s}\right]$-module injection

$$
0 \rightarrow M_{v h} \rightarrow M_{v}
$$

and a surjection

$$
M_{v} \rightarrow M_{v l} \rightarrow 0
$$

which are split over $R\left[\mathbf{X}, Y_{1}, \ldots, Y_{\sigma-1}\right] . H_{j}(\mathbf{X} ;)$ is an additive functor so that

$$
0 \rightarrow H_{j}\left(\mathbf{X} ; M_{v h}\right) \rightarrow H_{j}\left(\mathbf{X} ; M_{v}\right)
$$

and

$$
H_{j}\left(\mathbf{X} ; M_{v}\right) \rightarrow H_{j}\left(\mathbf{X} ; M_{v l}\right) \rightarrow 0
$$

are split over $R\left[Y_{1}, \ldots, Y_{s-1}\right]$ as well, thus verifying condition (iii).

TheOREM 5.2. Let $S=R\left[X_{1}, \ldots, X_{d}\right]$ be the polynomial ring in $d$ variables over a Noetherian ring $R$. Let $\mathscr{C}$ be a class of $S$-modules and let $\lambda$ be an additive function defined on $\mathscr{C}$ which takes on values in a totally ordered abelian group and assume $H_{i}\left(X_{s}, \cdots, X_{t} ; A\right) \in \mathscr{C}$ whenever $A \in \mathscr{C}, 0 \leq s \leq t \leq d$. Let $M \in \mathscr{C}$ be an $S$-module killed by a power of the irrelevant ideal $S_{+}=\left(X_{1}, \ldots, X_{d}\right)$ which admits $a$ high-low decomposition with respect to $X_{1}, \ldots, X_{d}$ and let

$$
\mathbf{x}_{1}, \ldots, \mathbf{x}_{n}=x_{11}, \ldots, x_{1 r_{1}}, x_{21}, \ldots, x_{n-1 r_{n-1}}, x_{n 1}, \ldots, x_{n r_{n}}
$$

be an ordered partition of $X_{1}, \ldots, X_{d}$. Then

$\lambda\left(H_{i_{n}}\left(\mathbf{x}_{n} ; \ldots ; H_{i_{2}}\left(\mathbf{x}_{2} ; H_{i_{1}}\left(\mathbf{x}_{1} ; M\right)\right) \ldots\right)\right) \geq \sum_{h\left(w_{n}\right)=i_{n}} \ldots \sum_{h\left(w_{1}\right)=i_{1}} \lambda\left(M_{w_{n} \cdots w_{2} w_{1}}\right)$ where $w_{\alpha} \in W_{r_{\alpha}}$.

Proof. The proof follows by induction on $n$ with the case of $n=1$ being a special case of Theorem 3.3. Suppose the statement holds for 
a partition consisting of $n-1$ sequences. The inductive hypothesis gives us

$$
\begin{aligned}
& \lambda\left(H_{i_{n}}\left(\mathbf{x}_{n} ; \ldots ; H_{i_{2}}\left(\mathbf{x}_{2} ; H_{i_{1}}\left(\mathbf{x}_{1} ; M\right)\right) \ldots\right)\right) \\
& \quad \geq \sum_{h\left(w_{n}\right)=i_{n}} \cdots \sum_{h\left(w_{2}\right)=i_{2}} \lambda\left(H_{i_{1}}\left(\mathbf{x}_{1} ; M_{w_{n} \cdots w_{2}}\right)\right) .
\end{aligned}
$$

The only question now is whether or not $H_{i_{1}}\left(\mathbf{x}_{1} ; M_{w_{n} \cdots w_{2}}\right)$ has a highlow decomposition with respect to $\mathbf{x}_{1}$ which, of course, it does by Lemma 5.1. Thus we get

$$
\begin{aligned}
\lambda\left(H_{i_{n}}\right. & \left.\left(\mathbf{x}_{n} ; \ldots ; H_{i_{2}}\left(\mathbf{x}_{2} ; H_{i_{1}}\left(\mathbf{x}_{1} ; M\right)\right) \ldots\right)\right) \\
& \geq \sum_{h\left(w_{n}\right)=i_{n}} \cdots \sum_{h\left(w_{1}\right)=i_{1}} \lambda\left(H_{0}\left(\varnothing ; M_{w_{n} \cdots w_{2} w_{1}}\right)\right) \\
& =\sum_{h\left(w_{n}\right)=i_{n}} \cdots \sum_{h\left(w_{1}\right)=i_{1}} \lambda\left(M_{w_{n} \cdots w_{2} w_{1}}\right) .
\end{aligned}
$$

REMARK 5.3. If $\lambda$ is positive on nonzero modules, for example length, then we have the deluxe form of Horrock's inequality

$$
\lambda\left(H_{i_{n}}\left(\mathbf{x}_{n} ; \ldots ; H_{i_{2}}\left(\mathbf{x}_{2} ; H_{i_{1}}\left(\mathbf{x}_{1} ; M\right)\right) \ldots\right)\right) \geq \prod_{j=1}^{n}\left(\begin{array}{c}
r_{j} \\
i_{j}
\end{array}\right) .
$$

REMARK 5.4. In the case of multi-graded modules, the requirement that the $\mathbf{x}, \ldots, \mathbf{x}_{n}$ be ordered can be dismissed outright since multigraded modules have high-low decompositions with respect to any ordering of the $X$ 's. However the partition requirement also turns out to be unnecessary.

Let $\mathbf{X}=X_{1}, \ldots, X_{r}$ and suppose that either $Y M=0$ or $Y$ occurs somewhere in $\mathbf{X}$. In either case the short exact sequence of Koszul complexes of $\S 4$ gives rise to the long exact sequence on homology

$$
\stackrel{Y}{\rightarrow} H_{i}(\mathbf{X} ; M) \stackrel{i_{*}}{\rightarrow} H_{i}(\mathbf{X}, Y ; M) \stackrel{q_{*}}{\rightarrow} H_{i-1}(\mathbf{X} ; M) \stackrel{Y}{\rightarrow} H_{i-1}(\mathbf{X} ; M) \rightarrow
$$

with the maps labeled $Y$ being equivalent to multiplication by zero. The resulting short exact sequence of Koszul complexes is actually split so that we obtain

$$
H_{i}(\mathbf{X}, Y ; M)=H_{i}(\mathbf{X} ; M) \oplus H_{i-1}(\mathbf{X}, M) .
$$

Combining these two reductions with our well-worn identity

$$
\left(\begin{array}{c}
s-1 \\
i
\end{array}\right)+\left(\begin{array}{l}
s-1 \\
i-1
\end{array}\right)=\left(\begin{array}{l}
s \\
i
\end{array}\right)
$$

allows us to get a much more general result in the multi-graded case. 
Theorem 5.5. Let $S=R\left[X_{1}, \ldots, X_{d}\right]$ be the polynomial ring in $d$ variables over a Noetherian base ring $R$. Let $\mathscr{C}$ be a class of $S$-modules and let $\lambda$ be an additive function defined on $\mathscr{C}$ which takes on values in a totally ordered abelian group and is positive on non-zero modules. Assume $H_{l}(\mathbf{X} ; A) \in \mathscr{C}$ whenever $A \in \mathscr{C}$ and $\mathbf{X}$ is a sequence of $X$ 's. Let $M$ be a finitely generated, multi-graded $S$-module killed by a power of the irrelevant ideal $S_{+}$. If

$$
\mathbf{x}_{1}, \ldots, \mathbf{x}_{n}=x_{11}, \ldots, x_{1 r_{1}}, x_{21}, \ldots, x_{n-1 r_{n-1}}, x_{n 1}, \ldots, x_{n r_{n}}
$$

is any (possibly redundant) sequence of $X$ 's, then

$$
\lambda\left(H_{i_{n}}\left(\mathbf{x}_{n} ; \ldots ; H_{i_{2}}\left(\mathbf{x}_{2} ; H_{i_{1}}\left(\mathbf{x}_{1} ; M\right)\right) \ldots\right)\right) \geq \prod_{j=1}^{n}\left(\begin{array}{c}
r_{j} \\
i_{j}
\end{array}\right) .
$$

Proof. Immediate from Remark 5.4.

Appendix. By omitting the multi-grading hypothesis, Theorem 5.5 becomes a new and much stronger conjecture. However, an unpublished example due to M. Hochster and J. McLaughlin shows this to be too much to hope for. We proceed with their example which produces a finite length module $M$ with $H_{0}\left(\mathbf{y} ; H_{1}(\mathbf{x} ; M)\right) \cong k$.

Let $V$ and $W$ be finite dimensional vector spaces of dimensions $n$ and $s$ respectively over a field $k$ and set $M=V \oplus W$. If $T \in$ $\operatorname{Hom}_{k}(V, W)$ is any linear transformation, it extends automatically to an endomorphism $T^{\prime} \in \operatorname{End}_{k} M$ by taking $T^{\prime}(v, w)=(0, T v)$. For any $S, T \in \operatorname{Hom}_{k}(V, W)$ we see that $S^{\prime} \circ T^{\prime}=T^{\prime} \circ S^{\prime}=0$. Let $R=k\left[X_{1}, \ldots, X_{d}\right]$. We can make $M$ into an $R$-module by choosing any $d$ linear transformations $T_{1}, \ldots, T_{d}$ and defining $X_{l} m=T_{i}^{\prime}(m)$. In particular we may take $d=n s$ and $T_{1}, \ldots, T_{d}$ to be a basis for $\operatorname{Hom}_{k}(V, W)$. In this setup $M$ is killed by the square of the irrelevant ideal $R_{+}$so we are free to think of $M$ as a module over $\widehat{R}=$ $k\left[\left[X_{1}, \ldots, X_{d}\right]\right]$.

Now let $e_{1}, \ldots, e_{n}$ be a basis for $V$ and set $U=\bigoplus_{1}^{n} V$. Set $e=$ $e_{1} \oplus \cdots \oplus e_{n} \in U$ and let $W=U / k e-a$ vector space of dimension $s=n^{2}-1$. If $j_{l}$ is the $i$ th inclusion of $V$ in $U$ and $q$ is the canonical surjection from $U$ to $W$, then $T_{i}=q j_{i}$ are linearly independent in $\operatorname{Hom}_{k}(V, W)$. Thus we may extend $T_{1}, \ldots, T_{n}$ to a basis $T_{1}, \ldots, T_{n s}$ for $\operatorname{Hom}_{k}(V, W)$ and $M=V \oplus W$ becomes a module over $R=$ $k\left[\left[X_{1}, \ldots, X_{n s}\right]\right]$ as described above. Since $R$ is local we have

length $M=\operatorname{dim}_{k} M=\operatorname{dim}_{k} V+\operatorname{dim}_{k} W=n+n^{2}-1$. 
We will show that $H_{1}=H_{1}\left(X_{1}, \ldots, X_{n} ; M\right)$ is a cyclic $R$-module. Thus $H_{0}\left(X_{1}, \ldots, X_{n s} ; H_{1}\right) \cong k$ in which case it is not true that

$$
l\left(H_{0}\left(X_{1}, \ldots, X_{n s} ; H_{1}\right)\right) \geq\left(\begin{array}{c}
n s \\
0
\end{array}\right)\left(\begin{array}{c}
n \\
1
\end{array}\right), \text { for } n \geq 1 .
$$

To find $H_{1}\left(X_{1}, \ldots, X_{n} ; M\right)$ we need to compute the homology of

$$
K_{2}\left(X_{1}, \ldots, X_{n} ; M\right) \stackrel{\alpha_{2}}{\rightarrow} K_{1}\left(X_{1}, \ldots, X_{n} ; M\right) \stackrel{\alpha_{1}}{\rightarrow} K_{0}\left(X_{1}, \ldots, X_{n} ; M\right) .
$$

Here we are just looking at

$$
M^{\left(\begin{array}{l}
n \\
2
\end{array}\right) \stackrel{\alpha_{2}}{\rightarrow}} M^{n} \stackrel{\alpha_{1}}{\rightarrow} M
$$

where $\alpha_{1}$ is just the column matrix

$$
\left(\begin{array}{c}
X_{1} \\
\vdots \\
X_{n}
\end{array}\right)
$$

operating on the right. Thus, in order to show that $H_{1}\left(X_{1}, \ldots, X_{n} ; M\right)$ is cyclic, it suffices to show that the module of relations on $X_{1}, \ldots, X_{n}$ with coefficients in $M$ is cyclic. $\left(m_{1}, \ldots, m_{n}\right) \in \operatorname{ker} \alpha_{1}$ means $\sum X_{i} m_{i}$ $=0$. If $m_{i}=v_{i} \oplus w_{i}$, this simply means $\sum T_{i} v_{i}=0$ in $W$, or $\left(v_{1}, \ldots, v_{n}\right)=c\left(e_{1}, \ldots, e_{n}\right)$ for some constant $c$ in $k$. Thus we have $\operatorname{ker} \alpha_{1}=k\left(e_{1}, \ldots, e_{n}\right)+W^{n}$ in $M^{n}$. We claim that $\left(e_{1}, \ldots, e_{n}\right) \in M^{n}$ is a generator for $\operatorname{ker} \alpha_{1}$ over $R$. Given $\left(w_{1}, \ldots, w_{n}\right) \in W^{n}$ and $c \in k$, there is a unique linear map $T$ in $\operatorname{Hom}_{k}(V, W)$ which takes $e_{i}$ to $w_{i}$. Since the action of every linear map $T$ in $\operatorname{Hom}_{k}(V, W)$ is represented by some linear form $X$ in $R$, we have

$$
c\left(e_{1}, \ldots, e_{n}\right)+\left(w_{1}, \ldots, w_{n}\right)=(c+X)\left(e_{1}, \ldots, e_{n}\right) .
$$

\section{REFERENCES}

[EG $\left.{ }_{1}\right]$ E. G. Evans and P. Griffith, Syzygies, London Math. Soc. Lecture Note Series, 106, Cambridge University Press, 1985.

[EG $\mathrm{EG}_{2}{ }_{-}$, Binomial behavior of Betti numbers for modules of finite length, Pacific J. Math., 133 (1988), 267-276.

[H] R. Hartshorne, Algebraic vector bundles on projection spaces: a problem list, Topology, 18 (1979), 117-128.

Received February 11, 1988. 


\section{PACIFIC JOURNAL OF MATHEMATICS EDITORS}

\author{
V. S. VARADARAJAN \\ (Managing Editor) \\ University of California \\ Los Angeles, CA 90024-1555-05 \\ Herbert Clemens \\ University of Utah \\ Salt Lake City, UT 84112 \\ ThOMAS ENRIGHT \\ University of California, San Diego \\ La Jolla, CA 92093
}

R. FINN

Stanford University

Stanford, CA 94305

HeRmann FlaschKa

University of Arizona

Tucson, AZ 85721

VAUGHAN F. R. JONES

University of California

Berkeley, CA 94720

Steven KerckHofF

Stanford University

Stanford, CA 94305
RoBION KIRBY

University of California

Berkeley, CA 94720

C. C. MOORE

University of California

Berkeley, CA 94720

Harold Stark

University of California, San Diego

La Jolla, CA 92093

\section{ASSOCIATE EDITORS}

\begin{tabular}{|c|c|c|c|c|}
\hline R. ARENS & $\begin{array}{l}\text { E. F. BECKENBACH } \\
(1906-1982)\end{array}$ & H. NeumanN & F. Wolf & K. YoshidA \\
\hline \multicolumn{5}{|c|}{ SUPPORTING INSTITUTIONS } \\
\hline \multicolumn{2}{|c|}{ UNIVERSITY OF ARIZONA } & \multicolumn{3}{|c|}{ UNIVERSITY OF OREGON } \\
\hline \multirow{2}{*}{\multicolumn{2}{|c|}{$\begin{array}{l}\text { UNIVERSITY OF BRITISH COLUMBIA } \\
\text { CALIFORNIA INSTITUTE OF TECHNOLOGY }\end{array}$}} & \multicolumn{3}{|c|}{ UNIVERSITY OF SOUTHERN CALIFORNIA } \\
\hline & & \multicolumn{3}{|c|}{ STANFORD UNIVERSITY } \\
\hline \multicolumn{2}{|c|}{ UNIVERSITY OF CALIFORNIA } & \multicolumn{3}{|c|}{ UNIVERSITY OF HAWAII } \\
\hline \multicolumn{2}{|c|}{ MONTANA STATE UNIVERSITY } & \multicolumn{3}{|c|}{ UNIVERSITY OF TOKYO } \\
\hline \multicolumn{2}{|c|}{ UNIVERSITY OF NEVADA, RENO } & \multicolumn{3}{|c|}{ UNIVERSITY OF UTAH } \\
\hline \multicolumn{2}{|c|}{ NEW MEXICO STATE UNIVERSITY } & \multicolumn{3}{|c|}{ WASHINGTON STATE UNIVERSITY } \\
\hline OREGON STA & UNIVERSITY & \multicolumn{3}{|c|}{ UNIVERSITY OF WASHINGTON } \\
\hline
\end{tabular}




\section{Pacific Journal of Mathematics}

\section{Vol. 141, No. $1 \quad$ November, 1990}

Yusuf Abu-Muhanna and Abdallah Khalil Lyzzaik, The boundary

behaviour of harmonic univalent maps $\ldots \ldots \ldots \ldots \ldots \ldots \ldots \ldots \ldots \ldots$

Lawrence Jay Corwin, Allen Moy and Paul J. Sally, Jr., Degrees and

formal degrees for division algebras and $\mathrm{GL}_{n}$ over a $p$-adic field $\ldots \ldots \ldots 21$

Ulrich Dierkes and Gerhard Huisken, The $n$-dimensional analogue of the

catenary: existence and nonexistence $\ldots \ldots \ldots \ldots \ldots \ldots \ldots \ldots 47$

Peter Gerard Dodds, C. B. Huijsmans and Bernardus de Pagter,

Characterizations of conditional expectation-type operators $\ldots \ldots \ldots \ldots 55$

Douglas Dokken and Robert Ellis, The Poisson flow associated with a

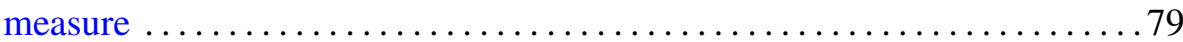

Larry J. Santoni, Horrocks' question for monomially graded modules . . . . 105

Zbigniew Slodkowski, Pseudoconvex classes of functions. II. Affine

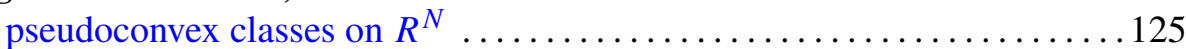

Dean Ellis Smith, On the Cohen-Macaulay property in commutative algebra

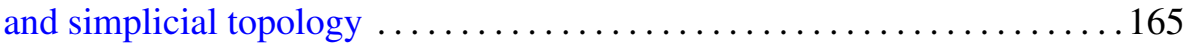

Michał Szurek and Jaroslaw Wisniewski, Fano bundles over $P^{3}$ and $Q_{3} \ldots 197$ 